\title{
Incorporating price in optimal product sampling for product diffusion*
}

\author{
Zhineng $\mathrm{Hu}$ \\ Uncertainty Decision-Making Laboratory \\ School of Administration and Management, Sichuan University, China 610065
}

(Received May 12 2006, Accepted September 1 2006)

\begin{abstract}
Firms use samples to increase the sales of almost all consumable goods. However, product sampling is also expensive. As a result, a firm needs to determine the optimal number of samples that must be available for trial by the innovators, early adopters, and other key consumers who influence the adoption rate of the new product. For this purpose, this paper builds the optimal models of product sampling by considering marketing strategies, especially focuses on the price strategy. Then the paper discusses the impact of the price strategy on the innovation diffusion of a new product with the help of estimated parameters, and gives out the meaningful explanation which can by used for marketing. Finally, the paper presents a future research framework on determining optimal product sampling for the market diffusion of a new product over time.
\end{abstract}

Keywords: New product, innovation diffusion, product sampling, marketing strategy

\section{Introduction}

Since publication of the Bass model, research on the modeling of the diffusion of innovations in marketing has resulted in an extensive literature. Contributions of this literature through past years are reviewed by $[24,26,38]$. This diffusion of an innovation traditionally has been defined as the process by which that innovation "is communicated through certain channels over time among the members of a social system"[23].

Following diffusion theory, new product adoption is viewed as a process consisting of two forces ${ }^{[24,38]}$.

(1) the external force, which consists of marketing efforts, such as advertising, price, etc.

(2) the internal force, comprised of interactions among consumers, such as word-of-mouth, imitation.

The Bass model and its extensions have been concerned with modeling the flow of consumers from the unaware to adoption states.

The fundamental diffusion model can be expressed as a differential equation ${ }^{[21]}$.

$$
\frac{\mathrm{d} N(t)}{\mathrm{d} t}=(a+b N(t))(\bar{N}-N(t))
$$

with the boundary condition $N\left(t=t_{0}\right)=N_{0}$, which is the number of the innovation product adopter at time $t_{0} ; N(t)$ is cumulative number of adopters at time $t ; \bar{N}(t)$ is total number of potential adopters in the social system at time $\mathrm{t} ; a$ and $b$ are coefficient of diffusion, i.e. external-influence coefficient and internal-influence coefficient, or innovation parameter and imitation parameter; $\frac{\mathrm{d} N(t)}{\mathrm{d} t}=S_{t}$ is the adoption rate, which is the number of new adopters at time $t$.

Equation (1) represents the diffusion process of durable-type innovation, i.e. the first-purchase model of new product. Thus, the quantity $\left(a+b N_{t}\right)$ is often called the diffusion rate whereas the quantity $\left(\bar{N}-N_{t}\right)$ is

\footnotetext{
* National Science Foundation for Distinguished Young Scholars, P. R. China (Grant No. 70425005) and the Teaching and Research Award Program for Outstanding Young Teachers in Higher Education Institutions of MOE of P. R. China (Grant No. 20023834-3). E-mail address: huzn@openmba.com.
} 
often designated by the untapped market or the saturation effect. An extensive model is the diffusion process of nondurable-type by considering repeated-purchase ${ }^{[24,26,38]}$. Obviously, those equations depict only the diffusion process of single product, and can be easily extended to depict the diffusion process of multipleinnovation products, such as competition-innovation diffusion model ${ }^{[2,8,20,22,31]}$. For above two category diffusion models, they both can be extended by considering multiple-unit adoptions ${ }^{[34]}$, seasonal factors of short lice cycle products ${ }^{[18]}$, stage diffusion model ${ }^{[39]}$, heterogeneous population ${ }^{[3,32]}$.

In the absence of a theoretical framework to provide guidelines for the incorporation of marketing mix variable in the diffusion process. Popular examples of studies that have incorporated one or more of the price, advertising, and distribution variables has been shown ${ }^{[27]}$. In that paper, the brief literature review suggests following results:

(1) marketing mix variables do affect the diffusion process;

(2) the way in which the marketing variables affect diffusion is basically an empirical equation;

(3) no concrete empirical evidence exists to support the assertion that multi-equation models are superior to single equation models in representing the diffusion process;

(4) there is a need to analyze further, theoretically and empirically, diffusion models that incorporate more than one marketing mix variable;

(5)there is an interest in examining more rigorously the robustness of policies derived form univariate diffusion models when additional marketing variables are included in the model.

Based on above review, in this paper, we focus on innovation diffusion of single product that incorporate price, because we are interesting in optimal product sampling of a new product.

Equation (1) represents the initial value condition indicating the number of adopters $N_{0}$ at the beginning of the diffusion process of the new product, i.e., at $t=0$. Traditionally, the value for $N_{0}$ has been assumed to be zero. In reality, $N_{0}>0$ can be taken as product sampling which can be an effective way to create an initial pool of "adopters", and this pool along with the regular group of innovators will influence other potential adopters via word-of-mouth ${ }^{[4,13]}$. However, sampling has not been extensively researched. It is typically considered as a tool to introduce consumers to a new product or to introduce a product to a new consumer group. [13] shows an approach for determining optimal product sampling for the diffusion of a new product, and analyzes the sampling with the supposition of unchanged market potential of innovation products and by not considering the impact of marketing strategies. Scott found that the effectiveness of sampling depends on the product price $^{[10]}$.

Thus, this paper study the sampling with the consideration of the impact of other marketing mix variables on the diffusion of a new product. References [16, 27, 38] show a comprehensive review of marketing mix variables, such as pricing, advertising, distribution and detailing. For simplicity, this paper only considers the impact of pricing strategies including skimming strategy and penetration strategy.

This paper is organized as follows. We formulate the theoretical models in section 2, and in section 3 discuss the optimal sampling strategy by numerical simulation. Finally, We discuss the managerial implications of our findings concluded in section 4 and discuss the future research framework on the modeling optimal product sampling.

\section{The models}

This section focuses on building the optimal model of product sampling for the durable-type innovation and nondurable-type innovation by considering marketing strategies.

\subsection{The models of sampling at time zero}

This subsection will present the models of sampling at time zero for the durable-type innovation and nondurable-type innovation by considering marketing strategy of pricing. 


\subsubsection{The first-purchase model}

In the product innovation context, diffusion models focus on the development of a life cycle curve and serve the purpose of forecasting first-purchase sales of innovations. That is, in the first-purchase diffusion models one assumes that, in the product planning horizon being considered, there are no repeat buyers and purchase volume per buyer is one unit. The number of adopters defines the unit sales for the product. Diffusion models, by definition, are concerned with representing the growth of a product category. Thus, the diffusion models incorporating price include following hypotheses:

(1) Diffusion of an innovation is independent of all other innovations.

(2) Nature of an innovation does not change over time.

(3) The geographic boundaries of the social system do not change over the diffusion process.

(4) The diffusion process is binary. The models assume that potential adopters of an innovation either adopt or do not adopt the innovation.

(5) Diffusion of an innovation is influenced by marketing strategy of pricing.

(6) Product and market characteristics do not influence diffusion patterns.

(7) There are no supply restrictions.

(8) There is only one adoption by adopting unit, and without repeat purchase.

(9) Sampling of the firm should be made at time zero.

Above hypotheses except the impact of marketing strategy of pricing are consistence with the hypotheses of Bass models. When launching a new product, a firm usually can choose between two distinct pricing strategies, i.e. market skimming and market penetration. A market-skimming strategy uses a high price initially to "skim" the market when the market is still developing. The market penetration strategy, in contrast, uses a low price initially to capture a large market share. The past diffusion models that incorporate price along assume that such a variable affects either the diffusion rate or the market potential. [30] proposes diffusion models for which either the coefficient of external influence alone is influenced by price or the coefficient of internal influence along is influenced by the same. [14] shows that price affects the diffusion rate (via the coefficients of external and internal influence). While [12] gives support to the assertion that price affects the market potential.

Thus, introduction of the impact of price in the Bass model framework generally has resulted in two types of normative pricing strategies. Studies derived from market skimming generally assume that price does not affect the population of potential adopters and produces a multiplicative effect on the rate of diffusion. That is, from equation (1),

$$
\frac{\mathrm{d} N_{t}}{\mathrm{~d} t}=\left(a+b N_{t}\right)\left(\bar{N}-N_{t}\right) g\left(p_{t}\right)
$$

where $g\left(p_{t}\right)$ is the price response function for the dynamic price $p_{t}$ at time $t$. Equation (2) assumes that price influences the rate of diffusion.

For market penetration strategy, we can see that some researchers have assumed that price affects the market potential. That is:

$$
\frac{\mathrm{d} N_{t}}{\mathrm{~d} t}=\left(a+b N_{t}\right)\left(\bar{N}\left(p_{t}\right)-N_{t}\right)
$$

where $\bar{N}\left(p_{t}\right)$ is the price response of $p_{t}$ at time $t$.

In equation (3), one can suppose $\overline{N_{t}}\left(p_{t}\right)=\overline{N_{t}} h\left(p_{t}\right)^{[27]}$. That is, Equation (3) is:

$$
\frac{\mathrm{d} N_{t}}{\mathrm{~d} t}=\left(a+b N_{t}\right)\left(\bar{N} h\left(p_{t}\right)-N_{t}\right)
$$

In equations (2) and (4), $p_{t}$ is real (deflated) price index for the average monthly basic rate. It is given by average monthly rate/consumer price index at time $t$ divided by the same quantity at time 1 , so that $p_{1}=1$. [27] gives the empirical response function of $\left(p_{t}\right)$ :

$$
g\left(p_{t}\right)=p_{t}^{-\eta}
$$

where $g>0, g^{\prime}<0, g^{\prime \prime}-\frac{2 g^{\prime 2}}{g}<0$. 
[13] shows product sampling may be accelerate the diffusion process of new products. The goal of product sampling for the firm is to initialize the diffusion process. The number of sampling may be too small to make diffusion process success, and the large number of sampling may be cost the products of the firm. So, for the durable-type innovation production, the goal of product sampling is to maximize the discounted cash flow (net present value) of new product:

$$
\max \pi=\int_{0}^{\infty} e^{-\rho t}\left(p_{t}-c\right) S_{t} \mathrm{~d} t-(h+c) N_{0}
$$

where $h$ is the cost of handling the sample per unit. This may include labor and material cost of wrapping, shipping and handling the sample, but it does not include the cost of production $c$. We also assume that the firm does not charge any price for the sample, i.e. the product is a giveaway, and assume that $S_{t}$ follows equation (2) or (4) and at time zero. So the problem is to find an optimal $N_{0}$ so as to maximize $\pi$. Therefore, we can conclude the optimal model of product sampling with various marketing strategies. The optimal model with penetration strategy of pricing is

$$
\left\{\begin{aligned}
\max \pi & =\int_{0}^{\infty} e^{-\rho t}\left(p_{t}-c\right) S_{t+1} \mathrm{~d} t-(h+c) N_{0} \\
\text { s.t. } & S_{t+1}=\left(a+b N_{t}\right)\left(\bar{N}-N_{t}\right) p_{t}^{-\eta}
\end{aligned}\right.
$$

where $N_{0}$ is the number of adopters of new product at time zero.

The optimal model with skimming strategy of pricing is

$$
\left\{\begin{aligned}
\max \pi & =\int_{0}^{\infty} e^{-\rho t}\left(p_{t}-c\right) S_{t+1} \mathrm{~d} t-(h+c) N_{0} \\
\text { s.t. } & S_{t+1}=\left(a+b N_{t}\right)\left(\bar{N} p_{t}^{-\eta}-N_{t}\right)
\end{aligned}\right.
$$

where $N_{0}$ is the number of adopters of new product at time zero.

Obviously, in equations (5) and (6), the price in state equations is real price index, and in objective function is real price, which is quite important in later numerical computation. Moreover, if $\eta \equiv 0$, we can obtain and optimal model of product sampling without marketing strategies ${ }^{[13]}$.

$$
\left\{\begin{aligned}
\max \pi & =\int_{0}^{\infty} e^{-\rho t}\left(p_{t}-c\right) S_{t+1} \mathrm{~d} t-(h+c) N_{0} \\
\text { s.t. } & S_{t+1}=\left(a+b N_{t}\right)\left(\bar{N}-N_{t}\right)
\end{aligned}\right.
$$

\subsubsection{The repeated-purchase model}

Equations (1), (3) and (4) have been developed to capture the adoption dynamics of a durable-type innovation production. There are nondurable products (such as ethical drugs) in which although word-of-mouth plays a critical role in generating the first-purchase adoption curve, the overall success of the product depends upon repeated adoptions. Thus, the diffusion models incorporating price include following hypotheses:

(1) Diffusion of an innovation is independent of all other innovations.

(2) Nature of an innovation does not change over time.

(3) The geographic boundaries of the social system do not change over the diffusion process.

(4) The diffusion process is binary. The models assume that potential adopters of an innovation either adopt or do not adopt the innovation.

(5) Diffusion of an innovation is influenced by marketing strategy of pricing.

(6) Product and market characteristics do not influence diffusion patterns.

(7) There are no supply restrictions.

(8) There is only one adoption by adopting unit, and with repeat purchase.

(9) Sampling of the firm should be made at time zero.

Above hypotheses except the impact of (6) and (8) are consistence with the hypotheses of Bass models. In this case, [19] assumes that for a nondurable product the total adoptions at any time $t$ are composed of the first-time adopters, given by equations (1), (3) or (4), and a certain percentage $r$ of the total number of current adopters. In the case of without marketing strategy, the state equation is 


$$
\frac{\mathrm{d} N(t)}{\mathrm{d} t}=(a+b N(t))[\bar{N}-N(t)]+r N(t-\tau)
$$

where $N\left(t=t_{0}\right)=N_{0}>0$ is the initial number of new product at time zero; $r$ is the rate of repeatedpurchase of new product; $\tau$ is the average life time of new product, or the lagged time for repeated-purchase of new product.

In this case, the firm maximizes the net present value given by

$$
\max \pi=\int_{0}^{\infty} e^{-\rho t}\left(p_{t}-c\right)\left(S_{t+1}+r N_{t}\right) \mathrm{d} t-(h+c) N_{0}
$$

where $h$ is the cost of handling the sample per unit; $c$ is the unit cost of the sample; $r$ is the percentage of the current number of adopters of new product, $r=0$ is for a durable-type innovation, $r>0$ is for a nondurabletype innovation. Then the model of the sample for nondurable-type innovation without any marketing strategy is

$$
\left\{\begin{aligned}
\max \pi & =\int_{0}^{\infty} e^{-\rho t}\left(p_{t}-c\right)\left(S_{t+1}+r N_{t}\right) \mathrm{d} t-(h+c) N_{0} \\
\text { s.t. } & S_{t+1}=\left(a+b N_{t}\right)\left(\bar{N}-N_{t}\right)+r N(t-\tau)
\end{aligned}\right.
$$

Equation (9) gives the optimal model of product sampling for nondurable-type innovation, which objective function is similar to one given by [13]. However, the state equation in that model lacks of $r N(t-\tau)$. Obviously, it is not properly built because that the goal of the optimal model is to determine the optimal level of the sample, but the state equation of new product is only for the first-purchase innovation. we will make further discussion for this idea in the section 3 .

\subsection{The extended models}

The models in previous subsection can be extended by considering mixed strategies, i.e. price, advertising, and distribution, or by considering the sampling over time, or by considering above twos together.

\subsubsection{The extended models incorporating mixed strategies}

In the case of with mixed marketing strategies, we can easily conclude the optimal model for nondurabletype innovation. Here, we only consider the extended models of sampling incorporating strategies of price, advertising, and distribution.

For the marketing variable of advertising, [1] assumes that advertising affect the coefficient of external influence. Upon an empirical discrimination among alternative model specifications using the diffusion data of new telephones in Germany, [33] concludes that advertising affects only the coefficient of internal influence.

For the marketing variable of distribution, [15] developes and gives empirical evidence to a lone diffusion model for which market potential is determined by the number of retailers that have adopted the new product.

Diffusion models that include more than one of the three marketing variables mentioned above are limited in number and are solely confined to the first two varibales - price and advertising. [37] proposes that price affects the rate of diffusion while advertising affects both the coefficients of external and internal influence in a linear fashion. Further, [27] characterizes qualitatively optimum price, advertising and distribution policies for new products, while repeat sales and possible entry of rivals are disregarded but discounting of future profit streams and a cost learning curve are allowed. Empirical research findings suggest that, for the considered case study, price affects the coefficient of innovation, advertising affects the diffusion rate, and distribution affects market potential.

Based on above review, we can conclude that the diffusion models incorporating strategies of price, adverting, and distribution include following hypotheses:

(1) Diffusion of an innovation is independent of all other innovations.

(2) Nature of an innovation does not change over time.

(3) The geographic boundaries of the social system do not change over the diffusion process.

(4) The diffusion process is binary. The models assume that potential adopters of an innovation either adopt or do not adopt the innovation. 
(5) Product and market characteristics do not influence diffusion patterns.

(6) There are no supply restrictions.

(7) There is only one adoption by adopting unit, and with repeat purchase.

(8) Diffusion of an innovation is influenced by marketing strategies of price, advertising, and distribution.

(9) Sampling of the firm should be made at time zero.

Thus, Following the ideas given by [25], we can present diffusion patterns of single product shown as figure 1.

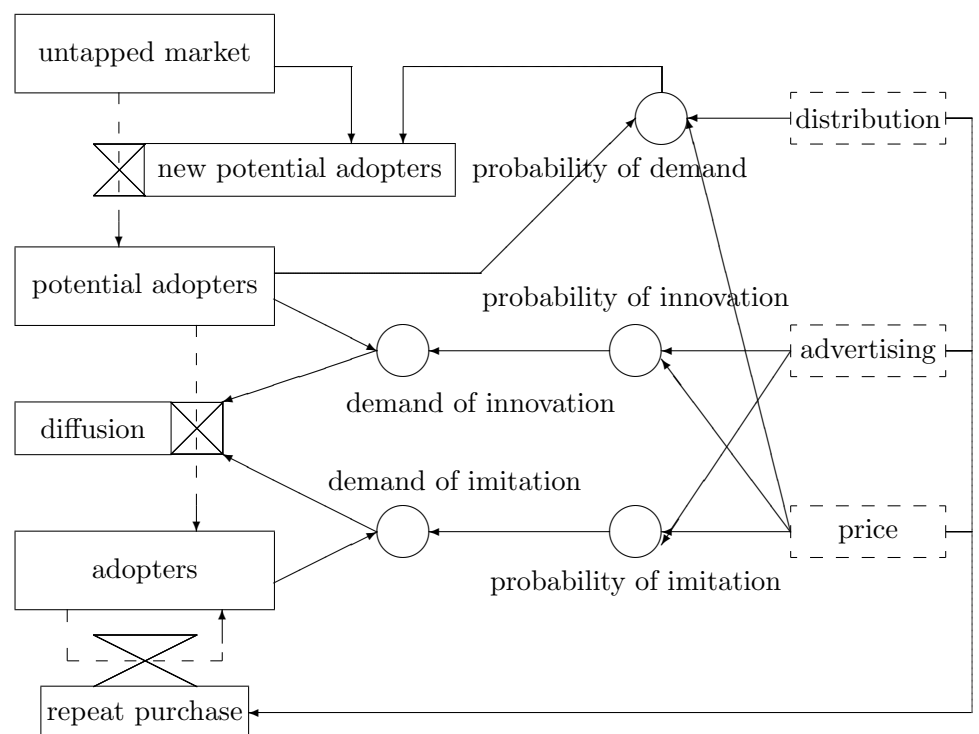

Fig. 1.

Based on Fig. 1 and discussion in [27], the price $(p)$ response response function $h(p)$, the advertising (u) efficiency function $q(u)$, and the distribution $(u)$ effectiveness function $w(D)$ are assumed to possess the following properties:

$$
h>0, q>0, w>0 ; h^{\prime}<0, q^{\prime}>0, w^{\prime}>0 ; h^{\prime \prime}-\frac{2 h^{\prime 2}}{h}<0, q^{\prime \prime}<0, w^{\prime \prime}>0
$$

Whenever a parameter or quantity in the diffusion model is assumed to depend on one or more of the marketing variables, this parmeter or quantity is simply multiplied, as appropriate, by one or more of the functions $h, q$ and $w$, see Tab. 1 .

Table 1. The impact of marketing strategies on diffusion process of a new product

\begin{tabular}{|c|c|c|c|}
\hline Strategy & Distribution & Advertising & Price \\
\hline Distribution & $\bar{N}$ & - & - \\
\hline Advertising & $\begin{array}{l}(a, \bar{N}) /(b, \bar{N}) / \\
(a+b N, \bar{N}) / \bar{N}\end{array}$ & $a / b / a+b N / \bar{N}$ & - \\
\hline Price & $\begin{array}{l}(a, \bar{N}) /(b, \bar{N}) / \\
(a+b N, \bar{N}) / \bar{N}\end{array}$ & $\begin{array}{c}a / b / a+b N / \bar{N} \\
(a, \bar{N}) /(b, \bar{N}) /(a+b N, \bar{N}) /(a, b) / \\
(\bar{N}, a) /(\bar{N}, b) /(\bar{N}, a+b N) /(b, a)\end{array}$ & $a / b / a+b N / \bar{N}$ \\
\hline \multicolumn{2}{|c|}{ Mixed strategies: } & $(a, b, \bar{N}) / \bar{N} /(b, a, \bar{N}) /(\underline{a}, \bar{N}) /(\underline{b}, \bar{N}$ & $(a+b N, \bar{N})$ \\
\hline
\end{tabular}

Then all of diffusion models including the first-purchase can be made in the Tab. $2 \sim 4$.

All of above diffusion models can be extended with repeat purchase in case of nondurable-type innovation. Thus, the general diffusion model incorporating marketing strategies is shown as follows: 
Table 2. The first-purchase models incorporating single marketing strategy

\begin{tabular}{c|ll}
\hline Marketing strategy & \multicolumn{2}{|l}{ First purchase model of new products: $\frac{\mathrm{d} N(t)}{\mathrm{d} t}=$} \\
\hline Price & $(a h+b N)(\bar{N}-N)$ & $(a+b h N)(\bar{N}-N)$ \\
& $(a+b N)(\bar{N}-N) h$ & $(a+b N)(\bar{N} h-N)$ \\
\hline Advertising & $(a q+b N)(\bar{N}-N)$ & $(a+b q N)(\bar{N}-N)$ \\
& $(a+b N)(\bar{N}-N) q$ & $(a+b N)(\bar{N} q-N)$ \\
\hline Distribution & $(a+b N)(\bar{N} w-N)$ & \\
\hline
\end{tabular}

Table 3. The first-purchase models incorporating two marketing strategies

\begin{tabular}{c|ll}
\hline Marketing strategy & \multicolumn{2}{|c}{ First purchase model of new products: $\frac{\mathrm{d} N(t)}{\mathrm{d} t}=$} \\
\hline Price/distribution & $(a h+b N)(\bar{N} w-N)$ & $(a+b h N)(\bar{N} w-N)$ \\
& $(a+b N)(\bar{N} w-N) h$ & $(a+b h N)(\bar{N} h w-N)$ \\
\hline Advertising/distribution & $(a q+b N)(\bar{N} w-N)$ & $(a+b q N)(\bar{N} w-N)$ \\
& $(a+b N)(\bar{N} w-N) q$ & $(a+b N)(\bar{N} q w-N)$ \\
\hline Price/advertising & $(a h+b N)(\bar{N} q-N)$ & $(a+b h N)(\bar{N} q-N)$ \\
& $(a+b N)(\bar{N} q-N) h$ & $(a h+b q N)(\bar{N} q-N)$ \\
& $(a h q+b N)(\bar{N}-N)$ & $(a+b h q N)(\bar{N}-N)$ \\
& $(a+b N)(\bar{N}-N) h q$ & $(a+b N)(\bar{N} h q-N)$ \\
& $(a q+b N)(\bar{N} h-N)$ & $(a+b q N)(\bar{N} h-N)$ \\
& $(a+b N)(\bar{N} h-N) q$ & $(a q+b h N)(\bar{N} h-N)$ \\
\hline
\end{tabular}

Table 4. The first-purchase models incorporating three marketing strategies

\begin{tabular}{c|ll}
\hline Marketing strategy & \multicolumn{2}{|c}{ First purchase model of new products: $\frac{\mathrm{d} N(t)}{\mathrm{d} t}=$} \\
\hline Price/advertising/distribution & $(a h+b q N)(\bar{N} w-N)$ & $(a q+b h N)(\bar{N} w-N)$ \\
& $(a h+b N)(\bar{N} h q w-N)$ & $(a h q+b N)(\bar{N} w-N)$ \\
& $(a+b h q N)(\bar{N} w-N)$ & $(a q+b N) h q(\bar{N} w-N)$ \\
\hline
\end{tabular}

$$
\frac{\mathrm{d} N(t)}{\mathrm{d} t}=f(N(t), a, b, \bar{N}, h, q, w)+r(h, q, w) N(t-\tau)
$$

where, $r=r(h, q, w)$ is the response function of $h, q$, w. when $r(h, q, w)=0$, the equation is reduced to the general model of first purchase.

Furthermore, when considering product growth models for medium-term forecasting of short life cycle products, we need introduce the seasonal influence parameter $\alpha_{t}{ }^{[18]}$. The general model can be extended as follows:

$$
\frac{\mathrm{d} N(t)}{\mathrm{d} t}=[f(N(t), a, b, \bar{N}, h, q, w)+r(h, q, w) N(t-\tau)] \alpha_{t}
$$

Then the general optimal model with strategies incorporating price, advertising and distribution is

$$
\left\{\begin{aligned}
\max \pi & =\int_{0}^{\infty} e^{-\rho t}\left(p_{t}-c\right) S_{t+1} \mathrm{~d} t-(h+c) N_{0} \\
\text { s.t. } & S_{t+1}=[f(N(t), a, b, \bar{N}, h, q, w)+r(h, q, w) N(t-\tau)] \alpha_{t}
\end{aligned}\right.
$$

where $N_{0}$ is the number of adopters of new product at time zero.

\subsubsection{The extended models over time}

Previous subsection 2.1 makes analysis of optimal product sampling at time zero for market diffusion of a new product. In fact, for all diffusion models, the free sample of new product $N_{0}$, is supposed to be sampling level at the begging of the diffusion process of new product. So the initial adopters of new product is 
$N_{0}=N\left(t_{0}\right)$. In a real market diffusion of a new product, the more cases will be happened is that the firm can make sampling freely over all time. Then we can take $N_{0}(t)$ as sampling at time $t$. For example, we suppose that last promotion time is $t=30$, which makes a special case result in above discussion in previous sections:

$$
N_{0}(0)=N_{0}, \quad N_{0}(1)=N_{0}(2)=\cdots=N_{0}(30)=0
$$

Of course, when considering the dynamical change of sampling at different promotion periods. The models in the section 2.1 will be changed, i.e. we have to consider the diffusion effect of sampling in previous periods when we make computation of the adopters at time $t$. Moreover, the state equation of the optimal models in section 2.1 can be extended to build more realistic models. In this way, we can make more realistic models with various pricing strategies, which can result in complexity analysis.

we can conclude that the diffusion models over time which incorporating strategies of price should include following hypotheses:

(1) Diffusion of an innovation is independent of all other innovations.

(2) Nature of an innovation does not change over time.

(3) The geographic boundaries of the social system do not change over the diffusion process.

(4) The diffusion process is binary. The models assume that potential adopters of an innovation either adopt or do not adopt the innovation.

(5) Product and market characteristics do not influence diffusion patterns.

(6) There are no supply restrictions.

(7) There is only one adoption by adopting unit, and with repeat purchase or not.

(8) Diffusion of an innovation is influenced by marketing strategies of price or not.

(9) Sampling of the firm should be made over time.

Based on above suppositions, a firm could distribute samples at different time during the whole product life to obtain the total optimal profit. The potential adopters at time $t$ are affected by adopters at time $(t-1)$, so does the diffusion process.

$$
S_{t}=\left\{a+b\left[N_{t-1}+N_{0}(t-1)\right]\right\}\left\{\bar{N}-\left[N_{t-1}+N_{0}(t-1)\right]\right\}
$$

Where $N_{0}(t-1)$ represents sampling amount at time $t-1, \bar{N}-\left[N_{t-1}+N_{0}(t-1)\right]$ denotes potential market level. Therefore, the term $a\left\{\bar{N}-\left[N_{t-1}+N_{0}(t-1)\right]\right\}$ denotes adoptions due to the external influence, and $b\left[N_{t-1}+N_{0}(t-1)\right]\left\{\bar{N}-\left[N_{t-1}+N_{0}(t-1)\right]\right\}$ denotes adoptions due to the internal influence.

When considering diffusion models of the sample over time, penetration pricing also affects on the potential market, the diffusion model is

$$
\begin{aligned}
S_{t} & =\left\{a+b\left[N_{t-1}+N_{0}(t-1)\right]\right\}\left\{\bar{N}-\left[N_{t-1}+N_{0}(t-1)\right]\right\} g\left(p_{t}\right) \\
& =\left\{a+b\left[N_{t-1}+N_{0}(t-1)\right]\right\}\left\{\bar{N}-\left[N_{t-1}+N_{0}(t-1)\right]\right\} p_{t}^{-\eta}
\end{aligned}
$$

For skimming pricing, the diffusion model of the sample over time is

$$
\begin{aligned}
S_{t} & =\left\{a+b\left[N_{t-1}+N_{0}(t-1)\right]\right\}\left\{\bar{N} h\left(p_{t}\right)-\left[N_{t-1}+N_{0}(t-1)\right]\right\} \\
& =\left\{a+b\left[N_{t-1}+N_{0}(t-1)\right]\right\}\left\{\bar{N} p_{t}^{-\eta}-\left[N_{t-1}+N_{0}(t-1)\right]\right\}
\end{aligned}
$$

For the model of the sampling over time, considering every possibility of sampling at different time during the product life, we get the objective function of the model of the sampling over time, which represents the maximization of net present value of the firm as follows.

$$
\max \pi=\int_{0}^{\infty} e^{-\rho t}\left[\left(p_{t}-c\right) s_{t}-(h+c) N_{0}(t)\right] d_{t}
$$

Where $\rho$ is discount rate, $p_{t}$ is price at time $t, h$ is unit handle cost, $c$ is unit cost, and $N_{0}(t)$ is the sampling at time $t$.

Therefore, the extended optimal model of the sample over time in the case of without marketing strategy is 


$$
\left\{\begin{aligned}
\max \pi & =\int_{0}^{\infty} e^{-\rho t}\left[\left(p_{t}-c\right) S_{t}-(h+c) N_{0}(t)\right] d t \\
\text { s.t. } & S_{t}=\left\{a+b\left[N_{t-1}+N_{0}(t-1)\right]\right\}\left\{\bar{N}-\left[N_{t-1}+N_{0}(t-1)\right]\right\}
\end{aligned}\right.
$$

Where $S_{0}=0$.

The extended optimal model of the sample over time in the case of with penetration pricing strategy is

$$
\left\{\begin{aligned}
\max \pi & =\int_{0}^{\infty} e^{-\rho t}\left[\left(p_{t}-c\right) S_{t}-(h+c) N_{0}(t)\right] d_{t} \\
\text { s.t. } & S_{t}=\left\{a+b\left[N_{t-1}+N_{0}(t-1)\right]\right\}\left\{\bar{N}-\left[N_{t-1}+N_{0}(t-1)\right]\right\} p_{t}^{-\eta}
\end{aligned}\right.
$$

Where $S_{0}=0$.

The extended optimal model of the sample over time in the case of with skimming pricing strategy is

$$
\left\{\begin{aligned}
\max \pi & =\int_{0}^{\infty} e^{-\rho t}\left[\left(p_{t}-c\right) S_{t}-(h+c) N_{0}(t)\right] d_{t} \\
\text { s.t. } & S_{t}=\left\{a+b\left[N_{t-1}+N_{0}(t-1)\right]\right\}\left\{\bar{N} p_{t}^{-\eta}-\left[N_{t-1}+N_{0}(t-1)\right]\right\}
\end{aligned}\right.
$$

Where $S_{0}=0$.

\section{Analysis of the model}

This section focuses on the numerical simulation computation for above optimal models of product sampling of new product.

\subsection{Analysis of sampling at time zero}

The first subsection shows supposition of the studied parameters, then the second subsection shows numerical analysis and results of sampling at time zero.

\subsubsection{Supposition of the studied parameters}

In reality, it is quite difficult to get analytical solutions for the optimal model (5) or (6). A better alternative is to solve the equations numerically with the values of parameters, such as $a, b, \bar{N}, \rho, p_{t}, c, h$ and $r$.

Firstly, it needs to discretize the objective functions in the optimal models as follows:

$$
Z=\sum_{t=0}^{30} \frac{1}{(1+r)^{t}}\left\{\left(p_{t}-c\right)\left(S_{t}+r N(t-1)\right)\right\}-(h+c) N_{0}
$$

Then given the values of the parameters, we calculate the cash flows for all percentage levels of maximal potentials of the samples to get the optimal results.

For durable-type innovation, we average the parameters given in the reference [21]. In the equation (1), the coefficient of external influence is 0.02 , the coefficient of internal influence is 0.35 , the market potential is 54 million $^{[13]}$. A metal-analysis of applications of diffusion models with 213 groups data in 15 papers shows ${ }^{[35]}$ : the analyses fit on the equation (1), the maximal coefficient of external influence is 0.23 , the minimal one is 0.00002 ; the maximal coefficient of external influence is 0.99 , the minimal one is 0.00003 . For an average view, the external coefficient of 213 applications is 0.03 , the internal one is 0.38 . Obviously, the divide of above internal coefficients by maximal potential is $b$ of the equation (1), also the one of this paper. Therefore, the priority is to give some parameters as follows:

(1) The coefficient of external influence $a=0.03$, maximal potential $\bar{N}=54$ million, the coefficient of internal influence $b=\frac{0.38}{\bar{N}}$.

The second class of the parameters is similar to ones of reference [13], as

(2) Discount rate $\rho=0.10$, the unit cost of the product $c=40$ dollar, the unit cost of the sample $h=10$ dollar.

When the sample does not influence the external coefficient, above two suppositions are needed to study the sample level of diffusion process of single product without market strategies. In the case of with marketing 
strategies, the change range of price elastics is from 1.9331 to 5.7439 . Then we also average it and give the third supposition as

(3) The elastic coefficient of price response function $\eta=3.8385$.

The suppositions $(1) \sim(3)$ are needed to study the sample level of diffusion process of single of new product when the free sample does not influence the external coefficient. In the case of discussing the impact of various price strategies on sample level, we suppose the goal price level of new product in the market is $\$$ 100 . Then in the case of skimming strategy, $p_{0}=\$ 140$ dollar, and the price is kept unchanged until $p_{5}=\$ 120$ which is unchanged again until $p_{10}=\$ 100$; In the case of penetration strategy of pricing: $p_{0}=60$ dollar, the price is unchanged until $p_{5}$ is up to 80 dollar, and which is unchanged until $p_{10}$ is up to 100 . Obviously, in the case of unchanged price strategy, $p_{t} \equiv 100$ in all time.

\subsubsection{Numerical analysis and results}

During the process of numerical simulation, the change range of $F_{0}$ is $0.1 \%$ every time. The algorithm is that we compute net present value of each percentage according to equation (20), and take the percentage corresponding to the maximal objective value as optimal value.

For three strategies of pricing depicted by equations $(5) \sim(7)$, the result is shown in Tab. $5^{2}$.

Table 5. The optimal sample level for durable-type innovation product

\begin{tabular}{cc|ccc}
\hline The strategy of pricing & Unchange pricing & Skimming & Penetration \\
Optimal value & strategy & strategy & strategy \\
\hline$F_{0}(\%)$ & $0.4 \%$ & $5.5 \%$ & 0 \\
NPV (million) & 16.314 & 24.891 & 10.382 \\
\hline
\end{tabular}

Based on the results in Tab. 5, the sampling level with the skimming strategy is the highest one among three strategies. In this case, the initial adopter is so small that the more sample is needed. And the market potential will be enlarged with the decreasing price. For the penetration strategy, the sampling level is the lowest one, but the diffusion speed is increasing with the constant market potential. If so, the firm should be careful of the marketing strategies with concurrent concrete market situation. Obviously, above discussions fit on the situation of real market. Unfortunately, the differences of optimal sampling among three strategies is too large for a stochastically choice of marketing strategies of the price. In the following discussion, we will make what-if analysis for constant pricing strategy, which can be easily extended to fit for other pricing strategies.

In the following, we discuss the impact of the sample in the case of different constant pricing. The price at initial time zero is quite low, so we make analysis for the price change range of $[40,200]$, the change of the sample with the price change is shown as Fig. 2. We can see that the sample reaches the zero and is always increasing when the price exceeds $\$ 90$, which causes the discussion of the price change range of $[40,800]$. In this case, the price always increases similarly. So, we choose the price change range of $[40,4000]$, the corresponding results can be seen in Fig. 2 and Fig. 3.

Obviously, the sample level does not to exceed 0.0960 when the price exceeds $\$ 3160$. Furthermore, we choose the price change of $[40,8000]$ to see the change of the sample, see the Fig. 4.

In the Fig. 4, the optimal sampling does not exceed 0.0980 in all cases. Again, we can discuss the sample change in an unusual case of the price change of $[40,80000]$, see the Fig. 5. In this case, the optimal sampling still under 0.0990. If so, we can gain a result that the optimal sampling is not to exceed 0.1 of market potential in all cases.

After the analysis of the impact of the price, we discuss the impact of internal factors or external factors on the diffusion process of new product. The coefficient of external factors can be changed from 0.00002 to 0.23 in reference [35], which change range is also taken as the coefficient change of external influence in our paper. The result of the Fig. 6 shows the impact of the change of external influence on the sample of

\footnotetext{
${ }^{2}$ The source code of results shown in this section can contact corresponding author.
} 


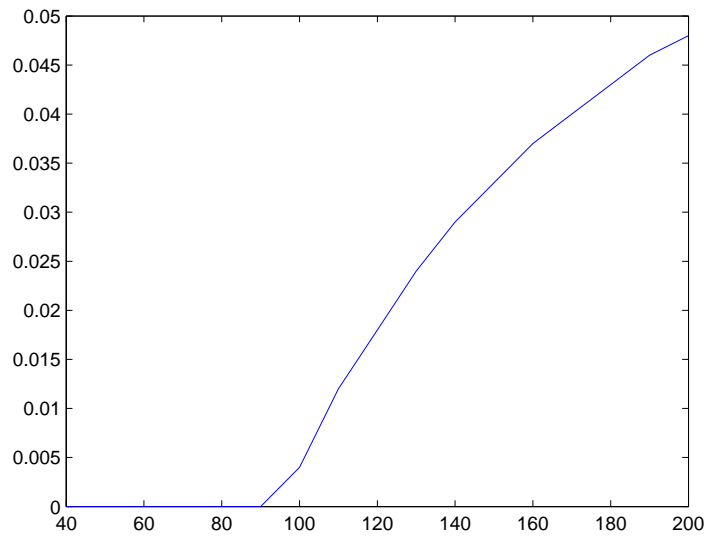

Fig. 2. The sample change with the price change of $[40,200]$

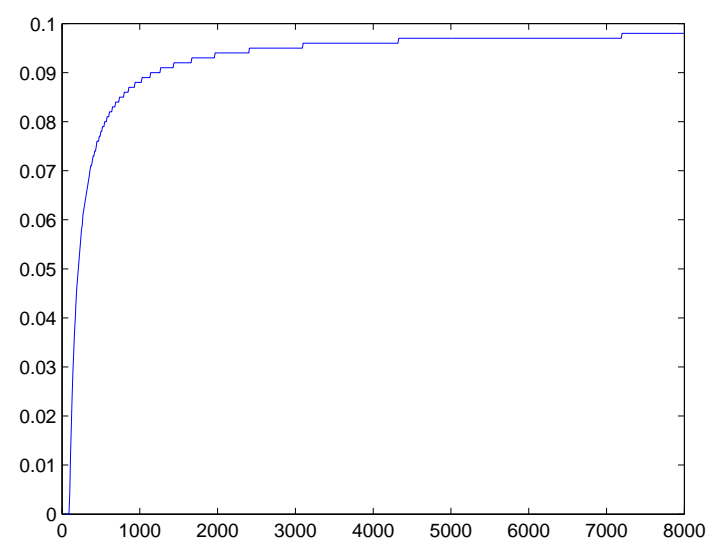

Fig. 4. The change of the sample with the price change of $[40,8000]$

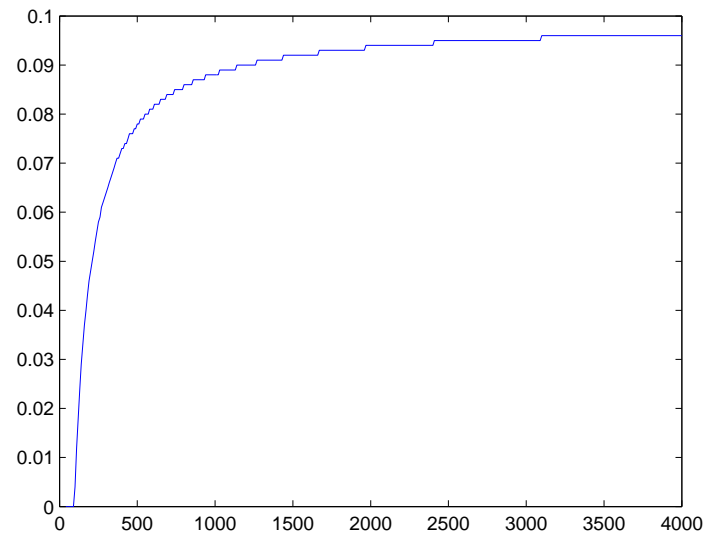

Fig. 3. The change of the sample with the price change of $[40,4000]$

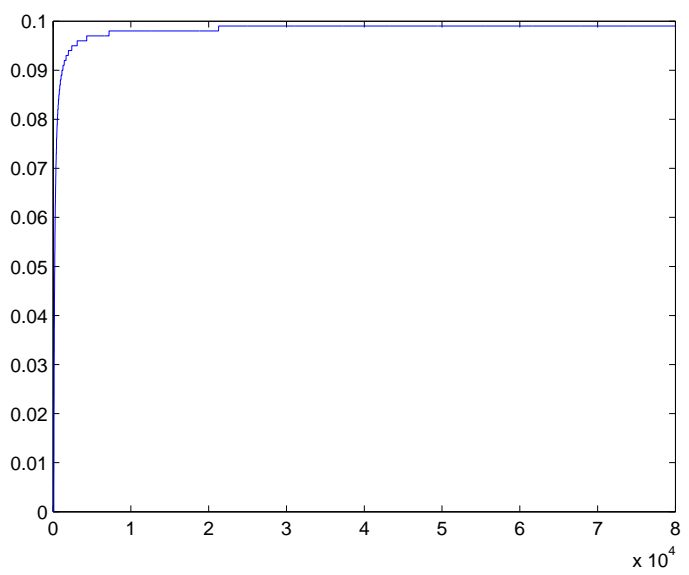

Fig. 5. The change of the sample with the price change of $[40,80000]$

new product. With the help of this result, one can see that the sampling level is always decreasing with the increasing coefficient of external influence, which is consistence with real diffusion process of new product.

The coefficient of internal influence can be changed from 0.00003 to $0.99^{[35]}$, therefore, which change range is also taken as the coefficient change of the internal influence in our paper. For the simplicity of the simulation, the change unit of internal influence is 0.00003 . Then the change of optimal sampling with the change of internal influence is shown in Fig. 7.

Based on the result of Fig. 7, one can see that the optimal sampling is always increasing with the increasing external influence, which is consistant with real diffusion process of new product.

In the case of constant pricing, we can easily make optimal sampling with different levels of repeatedpurchase for the diffusion of new product depicted as equation (9), which can be discussed in the case of with marketing strategies. Then we only show the method of discussing the impact of repeated-purchase on the innovation diffusion process of new product.

Obviously, the life time of the use of new product determines the lagged time of repeated-purchase without any marketing strategy. For different lagged time of nondurable-type product, the optimal sampling may be different in the case of equal repeated-purchase time. So, here we make discussion on the supposition of that the lagged time $\tau$ is 1,2,3 or 4, which corresponding optimal sampling of new product in the case of different repeated-purchase rate is shown in Fig. $8 \sim 11$.

With the results of above figures, one can see two obvious and meaningful managerial outcomes:

(1) The optimal sampling level increases with the increase of repeated-purchase rate of new product, however, this case will be reversed when the sampling exceeds the maximal value. 


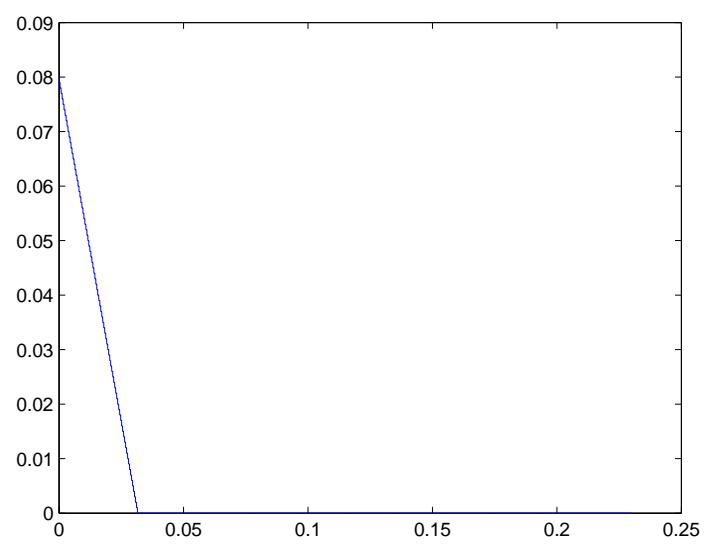

Fig. 6. The change of the sample in the case of the change of external influence

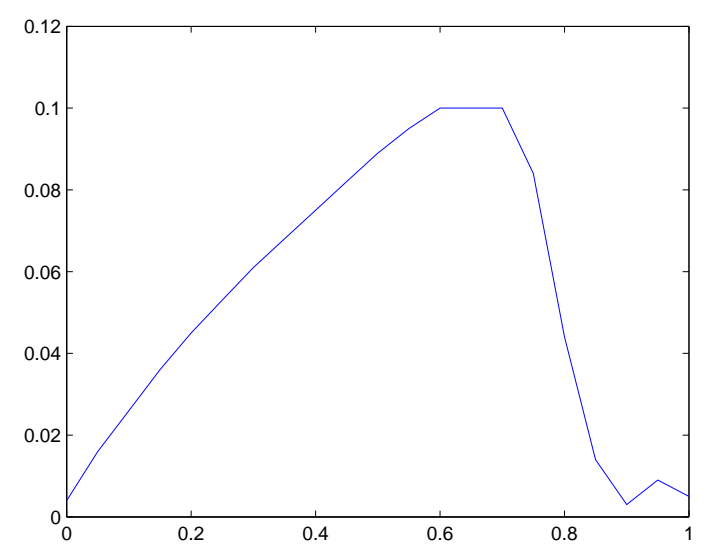

Fig. 8. The optimal sampling of new product when $\tau=1$

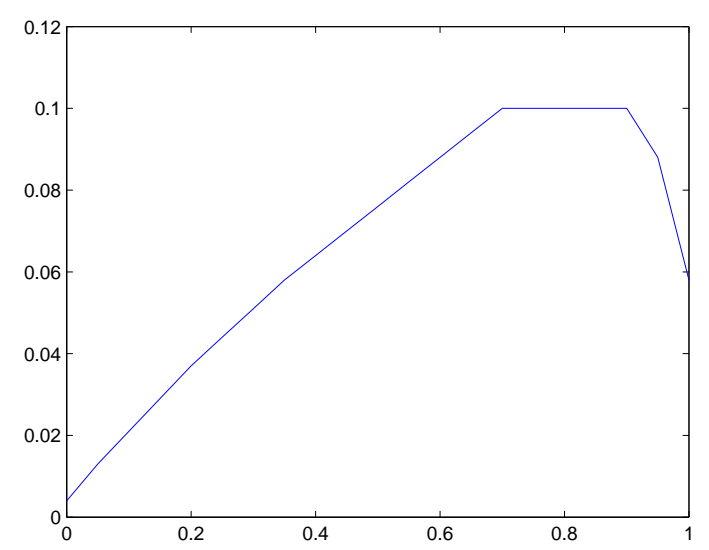

Fig. 10. The optimal sampling of new product when $\tau=3$

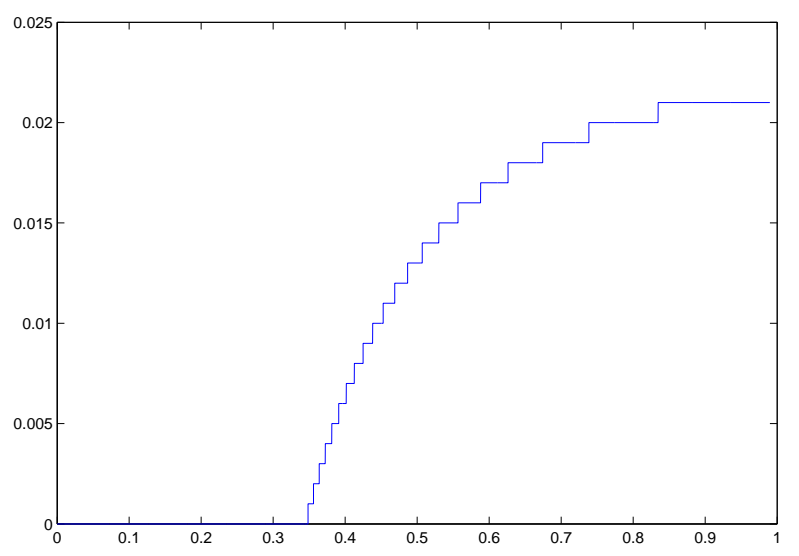

Fig. 7. The change of optimal sampling with the change of internal influence

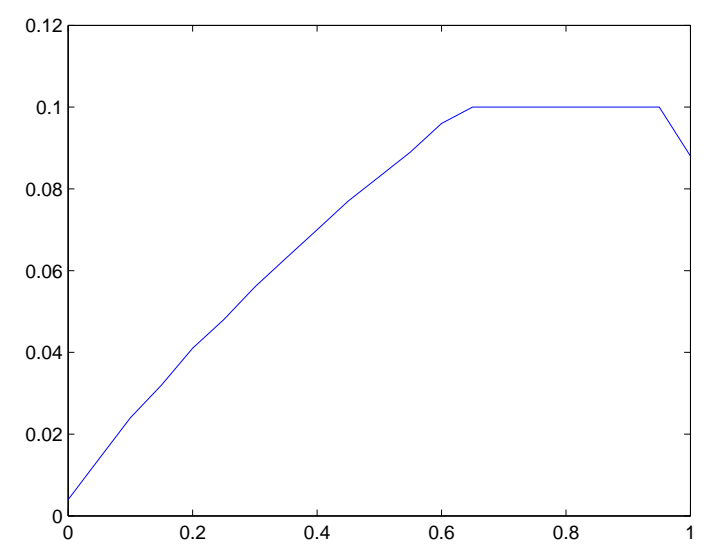

Fig. 9. The optimal sampling of new product when $\tau=2$

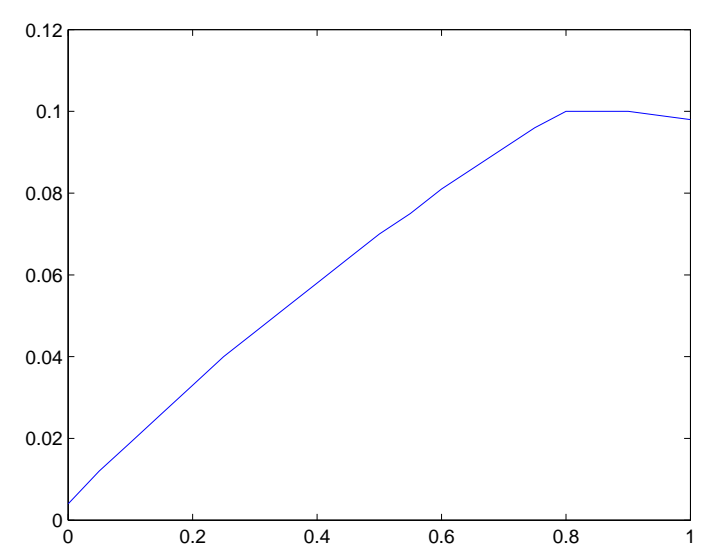

Fig. 11. The optimal sampling of new product when $\tau=4$

(2) The optimal sampling of new product will not be exceeded $10 \%$ of potential market with the help of previous analysis.

\subsection{Analysis of sampling over time}

The first subsection shows supposition of the studied parameters, Then the second subsection shows numerical analysis and results of sampling over time. 


\subsubsection{Supposition of the studied parameters}

We select first purchase model of durable product without pricing strategy to determine the optimal sampling level under a set of values for the parameters $a, b, \bar{N}, \rho, p, c$ and $h$.

Similarly, we select a finite time horizon of 30 periods to discrete objective function in the models (17).

$$
Z=\sum_{t=0}^{30} \frac{1}{(1+\rho)^{t}}\left[(p-c) S_{t}-(h+c) N_{0}(t)\right]
$$

Then we select a finite time horizon of 30 periods to discrete objective function in the model of repeatedpurchase, and determine the effect of repeat rate with a certain product life $\gamma$.

$$
Z=\sum_{t=0}^{30} \frac{1}{(1+\rho)^{t}}\left[(p-c)\left(S_{t}+r N_{t}\right)-(h+c) N_{0}(t)\right]
$$

When the parameters are characterized, the models of sampling over time are transformed to nonlinear programming models. For each parameter, Bass has figured out the average values of 11 kinds of durable product that external influence rate is 0.02 , internal influence rate is 0.35 and the max market potential is $\$ 54$ million. As an analysis of 213 groups of parameters, we get that the average external influence rate is 0.03 , internal influence rate is 0.38 , and the max market potential is $\$ 54$ million. Besides, the internal influence rate in (17) is the result of the internal influence rate above divided by the max market potential.

Thus, we set the parameters as $a=0.03, \bar{N}=5400000, b=\frac{0.38}{\bar{N}}$; and set $\rho=0.1, p=150, c=40$ and $h=10$ in a reasonable range.

\subsubsection{Numerical analysis and results}

For the optimal model of durable product without pricing strategy, we solve the optimal NPV and sampling level under the set of parameters above, and obtain the result as follows ${ }^{3}: Z=2.990242 E+08$, $N_{0}(1)=43628, N_{0}(2)=N_{0}(3)=\cdots=0 ; \operatorname{ratio}(1)=0.81 \%, \operatorname{ratio}(2)=\operatorname{ratio}(3)=\cdots=0$. Where $Z$ is optimal NPV, $N_{0}(t)$ is sampling amount at time $t$. $\operatorname{ratio}(t)$ is sampling level at time $t$, viz. $\operatorname{ratio}(t)=\frac{N_{0}(t)}{\bar{N}}$.

On the assumption of penetration pricing strategy, we set final market unit price is 15 . So the price is 100 at time zero, is kept unchange and up to 120 at time 5, and finally up to 150 at time 10 . In the diffusion model, the elasticity coefficient is set as $\eta=0.3835$. We solve the optimal NPV and sampling level and obtain the result as follows: $Z=2.203412 E+08, N_{0}(1)=N_{0}(2)=N_{0}(3)=\cdots=0$, ratio $(1)=\operatorname{ratio}(2)=$ ratio(3) $=\cdots=0$. Where $Z$ is the optimal NPV, $N_{0}(t)$ is sampling amount at time $t$. $\operatorname{ratio}(t)$ is sampling level at time $t$, viz. $\operatorname{ratio}(t)=\frac{N_{0}(t)}{\bar{N}}$.

On the assumption of penetration pricing strategy, we also assume the final price to be 150 . So the price is 200 at first, is kept unchange and falls to 170 at time 5, and finally falls to 150 at time 10 . And the elasticity coefficient is still set as $\eta=0.3835$. We solve the optimal NPV and sampling level and obtain the result as follows: $Z=3.960960 E+08, N_{0}(1)=310429, N_{0}(2)=N_{0}(3)=\cdots=0$; $\operatorname{ratio}(1)=5.75 \%$, $\operatorname{ratio}(1)=0.81 \%, \operatorname{ratio}(2)=\operatorname{ratio}(3)=\cdots=0$. Where $Z$ is optimal NPV, $N_{0}(t)$ is sampling amount at time $t$. $\operatorname{ratio}(t)$ is sampling level at time $t, \operatorname{viz}$. $\operatorname{ratio}(t)=\frac{N_{0}(t)}{\bar{N}}$.

The contrast analysis of optimal NPV and sampling level is summarized in Tab. 6.

Table 6. Optimal sampling level of the first-purchase model

\begin{tabular}{l|ccc}
\hline Marketing strategy & No pricing strategy & Penetration pricing strategy & Skimming pricing strategy \\
\hline Optimal NPV(unitYuan) & $2.990242 E+08$ & $2.203412 E+08$ & $3.960960 E+08$ \\
Sampling level ratio(1) & $0.81 \%$ & 0 & $5.75 \%$ \\
\hline
\end{tabular}

\footnotetext{
${ }^{3}$ The source code of results shown in this section can contact corresponding author.
} 
From Tab. 6, we can see that the sampling level with skimming pricing strategy is the highest, the second one is without pricing strategy and the lowest one is with penetration strategy. For skimming pricing strategy, the adoption is a little due to the high price. If we increase the samples, the diffusion of innovation product will be improved and the market potential will be affected by the trial adopters. Therefore, we could profit more at the beginning of diffusion of new product. For penetration pricing strategy, we will get a higher market share rate quickly for the lower price. So the objective at this time is to profit more by mass selling, and since the penetration pricing strategy has no effect on market potential, we should choose a lower sampling level.

As shown in Tab. 6, the optimal sampling are all happened at the beginning. Because the value we set for the parameters such as price is optional in a reasonable range, the difference between models on multipleperiods sampling and on initial sampling is not clear to understand. Thus, we have the specific effect of parameters as follows.

The impact on optimal sampling level of price, external influence rate and internal influence have been reviewed in [27]. From that we can see that the parameters influence the optimal level, especially price. Therefore, we analyze the specific effect on sampling level of price for durable product without pricing strategy.

In this case, we change the model of without pricing strategy by transforming the price as an external input coefficient. We get the result shown in Tab. 7, where $p$ is different price level and Ratio $(t)$ is sampling level at time $t$, viz. $\operatorname{Ratio}(t)=\frac{N_{0}(t)}{\bar{N}}$.

Table 7. Sampling level under different price level

\begin{tabular}{c|cccc}
\hline & Ratio(1) & Ratio(2) & Ratio(3) & Ratio(4) \\
\hline$p=100$ & 0 & 0 & 0 & 0 \\
$p=150$ & $0.81 \%$ & 0 & 0 & 0 \\
$p=200$ & $9.23 \%$ & 0 & 0 & 0 \\
$p=250$ & $15.43 \%$ & 0 & 0 & 0 \\
$p=300$ & $18.90 \%$ & $3.68 \%$ & 0 & 0 \\
$p=350$ & $21.62 \%$ & $7.09 \%$ & 0 & 0 \\
$p=400$ & $23.90 \%$ & $9.90 \%$ & 0 & 0 \\
$p=450$ & $25.83 \%$ & $12.23 \%$ & 0 & 0 \\
$p=500$ & $27.47 \%$ & $14.19 \%$ & 0 & 0 \\
$p=550$ & $28.75 \%$ & $15.69 \%$ & $1.12 \%$ & 0 \\
$p=600$ & $29.81 \%$ & $16.91 \%$ & $2.68 \%$ & 0 \\
$p=650$ & $30.76 \%$ & $17.99 \%$ & $4.04 \%$ & 0 \\
$p=700$ & $31.61 \%$ & $18.96 \%$ & $5.25 \%$ & 0 \\
$p=750$ & $32.37 \%$ & $19.83 \%$ & $6.32 \%$ & 0 \\
$p=800$ & $33.06 \%$ & $20.61 \%$ & $7.29 \%$ & 0 \\
$p=850$ & $33.68 \%$ & $21.32 \%$ & $8.15 \%$ & 0 \\
$p=900$ & $34.25 \%$ & $21.96 \%$ & $8.93 \%$ & 0 \\
$p=950$ & $34.77 \%$ & $22.55 \%$ & $9.64 \%$ & 0 \\
$p=1000$ & $35.25 \%$ & $23.09 \%$ & $10.29 \%$ & 0 \\
\hline
\end{tabular}

We can see from Tab. 7, the initial sampling level arises with a rising of price, and the periods increase with an increase in the value for the price. when the product cost is unchanged, we could profit more with higher unit price from unit product. So we need to increase samples to promote diffusion of innovation product and attract more potential consumers. If there's a big gap between price and cost, even if we sample a lot, we could still profit from adopters who buy our product at a high price level. So when price is increased, a firm would distribute more, which is consistent with practice phenomena.

In addition, if the price arises, the optimal samples are distributed at different time during the product life, which is quite different from ones on initial sampling. It is helpful to get feedback of innovation product, control the diffusion process of innovation product and profit more, which all shows the advantage of models on sampling over time. 
Then we promote the increment of price, and to determine the effect on sampling level of price more clearly. We solve the optimal sampling level with same parameters above, and obtain the result in Tab. 8, where $p$ is price, ratio(1) is initial sampling level, viz. $\operatorname{ratio}(1)=\frac{N_{0}(t)}{\bar{N}}$.

Table 8. Sampling level under different price value

\begin{tabular}{l|cccccccc}
\hline$p$ & 100 & 1000 & 2000 & 3000 & 4000 & 5000 & 6000 & 7000 \\
\hline Ratio(1) & $0.00 \%$ & $35.25 \%$ & $40.12 \%$ & $41.96 \%$ & $42.93 \%$ & $43.52 \%$ & $43.93 \%$ & $44.22 \%$ \\
\hline
\end{tabular}

For repeat-purchase model, we select optimal models without pricing strategy to determine the optimal sampling level and parameters analysis. For models with pricing strategy, it's easy to obtain the result by adding a certain parameter. It is shown that the repeat rate $r$ and product life cycle $\gamma$ influence the diffusion, which would have an effect on optimal sampling. We will analyze them respectively.

We set $\gamma=1$ and make repeat purchase rate as an input value. Then we get the result in Tab. 9, where $r b$ is repeat purchase rate and $\operatorname{Ratio}(t)$ is sampling level at time $t$, viz. $\operatorname{Ratio}(t)=\frac{N_{0}(t)}{\bar{N}}$.

Table 9. Sampling level with different repeat purchase rate

\begin{tabular}{c|ccccc}
\hline & Ratio(1) & Ratio(2) & Ratio(6) & Ratio(7) & ratio(8) \\
\hline$r b=0.0$ & $0.81 \%$ & 0 & 0 & 0 & 0 \\
$r b=0.1$ & $19.32 \%$ & $1.65 \%$ & 0 & 0 & 0 \\
$r b=0.2$ & $27.20 \%$ & $10.36 \%$ & 0 & 0 & 0 \\
$r b=0.3$ & $32.19 \%$ & $15.92 \%$ & 0 & 0 & 0 \\
$r b=0.4$ & $35.46 \%$ & $19.63 \%$ & 0 & 0 & 0 \\
$r b=0.5$ & $37.70 \%$ & $22.21 \%$ & 0 & 0 & 0 \\
$r b=0.6$ & $39.29 \%$ & $24.09 \%$ & 0 & 0 & 0 \\
$r b=0.7$ & $40.46 \%$ & $25.51 \%$ & 0 & 0 & $0.03 \%$ \\
$r b=0.8$ & $41.37 \%$ & $26.66 \%$ & 0 & $0.93 \%$ & 0 \\
$r b=0.9$ & $42.11 \%$ & $27.64 \%$ & 0 & $0.52 \%$ & 0 \\
$r b=1.0$ & $42.36 \%$ & $27.74 \%$ & $3.30 \%$ & 0 & 0 \\
\hline
\end{tabular}

we can see that the initial sampling level increase with an increase of repeat purchase rate, and the sampling elasticity is reduced, which is the same with the effect on sampling level of price in the first-purchase model.

Then, we set $\gamma=2, \gamma=3$ and solve the optimal initial sampling level and obtain the result in Tab. 10 and Tab. 11, where $r b$ is repeat purchase rate and ratio(1) is initial sampling level, viz. $\operatorname{ratio}(1)=\frac{N_{0}(t)}{\bar{N}}$.

Table 10. Initial sampling level with different repeat purchase rate when $\gamma=2$

\begin{tabular}{c|ccccccccccc}
\hline$r b$ & 0.0 & 0.1 & 0.2 & 0.3 & 0.4 & 0.5 & 0.6 & 0.7 & 0.8 & 0.9 & 1.0 \\
\hline Ratio(1) & $0.81 \%$ & $18.96 \%$ & $26.85 \%$ & $31.90 \%$ & $35.00 \%$ & $37.21 \%$ & $38.83 \%$ & $40.04 \%$ & $40.98 \%$ & $41.71 \%$ & $42.29 \%$ \\
\hline
\end{tabular}

Table 11. Initial sampling level with different repeat purchase rate when $\gamma=3$

\begin{tabular}{c|ccccccccccc}
\hline$r b$ & 0.0 & 0.1 & 0.2 & 0.3 & 0.4 & 0.5 & 0.6 & 0.7 & 0.8 & 0.9 & 1.0 \\
\hline Ratio(1) & $0.81 \%$ & $18.68 \%$ & $26.51 \%$ & $31.46 \%$ & $34.56 \%$ & $36.80 \%$ & $38.45 \%$ & $39.70 \%$ & $40.66 \%$ & $41.40 \%$ & $42.01 \%$ \\
\hline
\end{tabular}

we can see the same trend of initial sampling level which shows that the sampling level increase with an increase of repeat purchase rate. The sampling level of nondurable product is higher than durable product due to the effect of repeat purchase rate. On the sampling over time, the sampling of in the first-purchase model 
is up to $35.25 \%$ as the price up to 1000 , while in the repeat purchase model, if the repeat purchase rate is 0.4 , we could get $35 \%$ optimal sampling level with lower price 150 .

In addition, we determine the effect of product life cycle $\gamma$ with a certain repeat purchase rate. Based on above results, we contrast sampling level of product life $\gamma=1, \gamma=2$, and $\gamma=3$ shown in Tab. 12.

Table 12. Initial sampling level under same repeat purchase rate

\begin{tabular}{c|ccccccccccc}
\hline$r b$ & 0 & 0.1 & 0.2 & 0.3 & 0.4 & 0.5 & 0.6 & 0.7 & 0.8 & 0.9 & 1.0 \\
\hline$\gamma=1$ & $0.81 \%$ & $19.32 \%$ & $27.20 \%$ & $32.19 \%$ & $35.46 \%$ & $37.70 \%$ & $39.29 \%$ & $40.46 \%$ & $41.37 \%$ & $42.11 \%$ & $42.36 \%$ \\
$\gamma=2$ & $0.81 \%$ & $18.96 \%$ & $26.85 \%$ & $31.90 \%$ & $35.00 \%$ & $37.21 \%$ & $38.83 \%$ & $40.04 \%$ & $40.98 \%$ & $41.71 \%$ & $42.29 \%$ \\
$\gamma=3$ & $0.81 \%$ & $18.68 \%$ & $26.51 \%$ & $31.46 \%$ & $34.56 \%$ & $36.80 \%$ & $38.45 \%$ & $39.70 \%$ & $40.66 \%$ & $41.40 \%$ & $42.01 \%$ \\
\hline
\end{tabular}

As shown in Tab. 12 lengthways, under the same repeat purchase rate, the innovation product, which has longer product life cycle, has a lower sampling level. In previous subsection, we can also see that if the product life cycle become longer, the diffusion increment unit will decrease more. Given a certain price level, it's necessary to profit more by fewer samples.

For analysis of repeat purchase model, we can modify same parameters accordingly to get the optimal sampling level under different conditions. So in this paper we will not analyze it in full detail.

\section{Concluding remarks}

Then present paper is the only model study of incorporating price in product sampling, which extends the discussion of pioneer work of the sample by [13]. We get many managerial results, in which, the most meaningful ones are the trend of sampling when considering repeated-purchase, and the maximal sampling when considering sampling at time zero. In addition, a question now is how can one use these in a practical setting. The implementation of previous analytical results will involve the following steps ${ }^{[13]}$.

(1) Identification of the diffusion parameters including the coefficient of innovation, coefficient of imitation, and market potential. In the case of having no historic data or seldom ones, we need to make estimation with the help of sales judgers. A better choice is to make a meta-analysis of previous diffusion models ${ }^{[35]}$.

(2) Specification of the discount rate by account department.

(3) Determination of the price of the product by marketing department. Generally, we need to compute the deflated price index ${ }^{[27]}$.

(4) Estimation of the product's unit cost and handling cost by production department and marketing department.

(5) Substitution of the various parameter values in the analytical formulation to obtain the optimal sampling levels, which is discussed carefully in our paper.

Among above steps, the most difficult one is the first step which involves parameter estimation considerations of various diffusion models, see the references ${ }^{[24,28,38]}$.

In the future research work, whenever by considering the sample at time zero or over time, the models of present paper can be extended to determine the optimal product sampling in the case of expanding the hypotheses in section 2.

(1) Diffusion of an innovation is dependent of other innovations including competition innovation diffusion $^{[8,22,31]}$, complementary innovation diffusion, contingent innovation diffusion.

(2) The geographic boundaries of the social system is changed over the diffusion process, such as space diffusion studied with the method of Cellular Automata Modeling $[6,7,9,29,36]$.

(3) The diffusion process is divided into three stages, which models assume that consumers include the unknowns, potential adopters and adopter ${ }^{[24,39]}$.

(4) Product and market characteristics influence diffusion patterns, such as heterogeneous population $^{[3,32]}$.

(5) There are supply restrictions ${ }^{[5,11,17]}$.

(6) There is multi-adoption by adopting unit, and with repeat purchase ${ }^{[34]}$. 


\section{References}

[1] D. J. A., E. Muller. Models for new prduct diffusion through advertising and word-of-mouth. Management Science, 1978, 24: 1568-1578.

[2] A. P. Bassanini, G. Dosi. Competing technologies, international diffusion and the rate of convergence to a stable market structure andrea p. bassanini. Tech. Rep., International Institute for Applied Systems Analysis, 1998.

[3] R. Chatterjee, J. Eliashberg. The innovatioh diffusion process in a heterogeneous population: A micromodeling approach. Management Science, 1990, 36: 1057-1079.

[4] DavidE.Sprott, T. A. Shimp. Using product sampling to augment the perceived quality of store brands. Journal of Retailing, 2004, 80: 305-315.

[5] R. T. Frambach, H. G. Barkema, B. Nooteboom, M. Wedel. Adoption of a service innovation in the business market: An empirical test of supply-side variables. Journal of Business Research, 1998, 41: 161-174.

[6] M. A. Fuentes, M. N. Kuperman. Cellular automata and epidemiological models with spatial dependence. Physica A, 1999, 267: 471-486.

[7] H. Fuk, N. Boccara. Cellular automata models for diffusion of innovations, 2002.

[8] M. Givon, V. Mahajan, E. Muller. Assessing the relationship between the user-based market share and unit salesbased market share for pirated software brands in competitive markets. Technological Forecasting and Social Change, 1997, 55: 131-144.

[9] J. Goldenberg, S. Efroni. Using cellular automata modeling of the emergence of innovations. Technological Forecasting \& Social Change, 2001, 68: 293-308.

[10] A. Heiman, B. Mcwilliams, Z. Shen, D. Zilberman. Learning and forgetting: Modeling optimal product sampling over time. Management Science, 2001, 47(4): 532-546.

[11] T.-H. Ho, S. Savin, C. Terwiesch. Managing demand and sales dynamics in new product diffusion under supply constraint. Management Science, 2002, 48(2): 187-206.

[12] D. Horsky. A diffusion model incorporating product benefits, price, income, and information. Marketing Science, 1990, 9: 342-365.

[13] D. Jain, V. Mahajan, E. Muller. An approach for determining optimal product sampling for the diffusion of a new product. Journal of Production Innovation Management, 1995, 12: 124-135.

[14] D. C. Jain, R. C. Rao. Effect of price on the demand for durables: Modeling, estimation and findings. Journal of Business and Economic Statisitics, 1990, 8: 163-170.

[15] J. M. Jones, C. J. Ritz. Incorporating distribution into new product diffusion models. Int. Res. in Marketing, 1991, 8: $91-112$.

[16] S. Kalish, V. Mahajan, E. Muller. Waterfall and sprinkler new-product strategies in competitive global markets. International Journal of Research in Marketing, 1995, 12: 105-119.

[17] S. Kumar, J. M. Swaminathan. Diffusion of innovations under supply constraints. Operations research, 2003, 51(6): 866-879.

[18] A. A. Kurawarwala, H. Matsuo. Product growth models for medium-term forecasting of short life cycle products. Technological Forecasting and Social Change, 1998, 57: 169-196.

[19] L. G. L., A. G. Rao, S. Kalish. Bayesian estimation and control of detailing effort in a repeat-purchase diffusion environment. Management Science, 1981, 27: 493-506.

[20] C. H. Loch, B. A. Huberman. A punctuated-equilibrium model of technology diffusion. Management Science, 1999, 45(2): 160-170.

[21] B. F. M. A new product growth model for consumer durables. Management Science, 1969, 15: $215-227$.

[22] G. M., M. V., M. E. Software piracy: Estimation of lost sales and the impact on software diffusion. Journal of Marketing, 1995, 59(1): 29-37.

[23] R. E. M. Diffusion of Innovations. Free Press, New York, 1995.

[24] V. Mahajan, R. A. Peterson. Models for innovation diffusion. in: Sage University Paper Series on Quantiative Applications in the Social Sciences Volume 48, Sage Publications, Beverly Hills and London, 1985.

[25] F. H. Maier. New product diffusion models for decision support in innovation management, 1997. Arbeitspapier aus dem Industrieseminar der Universität Mannheim, Mannheim.

[26] N. Meade, T. Islam. Modelling and forecasting the diffusion of innovation - a 25-yearre view. Internaltional Journal of forecasting, 2006, 22: 519-545.

[27] H. I. Mesak. Incorporating price, advertising and distribution in diffusion models of innovation: Some theoretical and empirical results. Computers Ops. Res., 1996, 23(10): 1007-1023.

[28] H. I. Mesak, J. W. Clark. Monopolist optimum pricing and advertising policies for diffusion models of new product innovations. Optimal Control Applications \& Methods, 1998, 19: 111-136.

[29] S. Moldovan, J. Goldenberg. Cellular automata modeling of resistance to innvations: Effects and solutions. Technological Forecasting \& Social Change, 2004, 71: 425-442. 
[30] P. M. Parker. Price elasticity dynamics over the adoption life cycle. Journal of Marketing Research, 1992, 24: 358-367.

[31] A. Prasad, V. Mahajan. How many pirates should a software firm tolerate? an analysis of piracy protection on the diffusio of software. International Journal of research in Marketing, 2003, 20: 337-353.

[32] S. P. R., M. D. P. A mathematical model for new product diffusion: The influence of innovators and imitators. Math Comput Modelling, 1992, 16(4): 11-26.

[33] H. Simon, K. H. Sebastian. Diffusion and advertising: The german telephone company. Management Science, 1987, 33: 451-466.

[34] P. R. Steffens. A model of multiple-unit ownership as a diffusion process. Technological Forecasting \& Social Change, 2003, 70: 901-917.

[35] F. Sultan, J. U. Farely, D. R. Lehmann. A meta-analysis of applications of diffusion models. Journal of Marketing Research, 1990, 27: 70-77.

[36] V. Terrier. Two-dimensional cellular automata and their nerghborhoods. Theoretical Computer Science, 2004, 312: 203-222.

[37] G. L. Thompson, J. T. Teng. Optimal pricing and advertising policies ofr new product oligopoly models. Marketing Science, 1984, 3: 148-168.

[38] M. V., E. Muller, F. M. Bass. New product diffusion models in marketing: A review and directions for research. Journal of Marketing, 1990, 54(1): 1-26.

[39] W. Wang, P. Fergola, S. Lombardo, G. Mulone. Mathematical models of innovation diffusion with stage structure. Applied Mathematical Modelling, 2006, 30: 129-146. 